\title{
Effect of editors' implementation of CONSORT guidelines on the reporting of abstracts in high impact medical journals: interrupted time series analysis
}

\author{
(c) $(1)$ (8) OPEN ACCESS
}

\author{
Sally Hopewell senior research fellow ${ }^{12}$, Philippe Ravaud professor ${ }^{2345}$, Gabriel Baron statistician ${ }^{234}$, \\ Isabelle Boutron associate professor ${ }^{2345}$
}

${ }^{1}$ Centre for Statistics in Medicine, University of Oxford, Wolfson College, Oxford OX2 6UD, UK; ${ }^{2}$ INSERM, U738, Paris, France ; ${ }^{3}$ Assistance Publique des Hôpitaux de Paris, Hôtel Dieu Hospital, Centre of Clinical Epidemiology, Paris; ${ }^{4}$ University of Paris Descartes, Sorbonne Paris Cité, Faculty of Medicine, Paris; ${ }^{5}$ French Cochrane Centre, Paris

\begin{abstract}
Objective To investigate the effect of the CONSORT for Abstracts guidelines, and different editorial policies used by five leading general medical journals to implement the guidelines, on the reporting quality of abstracts of randomised trials.

Design Interrupted time series analysis.

Sample We randomly selected up to 60 primary reports of randomised trials per journal per year from five high impact, general medical journals in 2006-09, if indexed in PubMed with an electronic abstract. We excluded reports that did not include an electronic abstract, and any secondary trial publications or economic analyses. We classified journals in three categories: those not mentioning the guidelines in their instructions to authors (JAMA and New England Journal of Medicine), those referring to the guidelines in their instructions to authors but with no specific policy to implement them (BMJ), and those referring to the guidelines in their instructions to authors with an active policy to implement them (Annals of Internal Medicine and Lancet). Two authors extracted data independently using the CONSORT for Abstracts checklist.
\end{abstract}

Main outcome Mean number of CONSORT items reported in selected abstracts, among nine items reported in fewer than $50 \%$ of the abstracts published across the five journals in 2006.

Results We assessed 955 reports of abstracts of randomised trials. Journals with an active policy to enforce the guidelines showed an immediate increase in the level of mean number of items reported (increase of 1.50 items; $P=0.0037$ ). At 23 months after publication of the guidelines, the mean number of items reported per abstract for the primary outcome was 5.41 of nine items, a $53 \%$ increase compared with the expected level estimated on the basis of pre-intervention trends. The change in level or trend did not increase in journals with no policy to enforce the guidelines (BMJ, JAMA, and New England Journal of Medicine).

Conclusion Active implementation of the CONSORT for Abstracts guidelines by journals can lead to improvements in the reporting of abstracts of randomised trials.

\section{Introduction}

Clear, transparent, and sufficiently detailed abstracts of journal articles reporting randomised trials are important, because readers often base their initial assessments of a trial on the content of the abstract. In some cases, health practitioners will have access only to the abstract, and could, therefore, make healthcare decisions based solely on the information in that abstract. ${ }^{1}$ As such, the journal abstract should be a clear and accurate reflection of what is included in the journal article. However, several studies have highlighted problems in the accuracy and quality of abstracts, including a lack of information about the trial methodology and the robustness of the trial results. ${ }^{2}$ Studies comparing the accuracy of information reported in a journal abstract with that reported in the text of the full publication have found claims that are inconsistent with, or missing from, the body of the full article. ${ }^{45}$ This inadequate

Correspondence to: S Hopewell sally.hopewell@csm.ox.ac.uk

Extra material supplied by the author (see http://www.bmj.com/content/344/bmj.e4178?tab=related\#webextra)

Web table 1: Reporting of CONSORT for Abstracts items across journals in 2006

Web appendix 1: Segmented regression with one group-model to get an estimate of the difference between pre and post intervention Web table 2: Parameter estimates from segmented regression models predicting the mean monthly number of items reported per abstract Web table 3: Reporting of CONSORT for Abstracts items per journal in (A) 2006, (B), 2007, (C) 2008, and (D) 2009

Web appendix 2: Change in the mean number of items reported per abstract for primary outcome (0-9) over time (from January 2006 to December 2009) before and after the introduction of the CONSORT for Abstracts guidelines implemented in January 2008 by journal 
reporting could seriously mislead a reader's interpretation of the trial findings. ${ }^{6}$

In response to these limitations, an extension to the CONSORT statement (CONsolidated Standards Of Reporting Trials) was published in January 2008, providing a list of essential items to include when reporting the main results of a randomised trial in a journal or conference abstract. ${ }^{78}$ However, the effect of the CONSORT for Abstracts guidelines has not been widely evaluated. In this study, we aimed to investigate the effect of these guidelines, and journals' editorial policy to implement them, on the reporting quality of abstracts of randomised trials published.

\section{Methods \\ Design and sample}

To assess the effect of publication of the CONSORT for Abstracts guidelines, we planned a segmented regression of interrupted time series analyses, a quasi-experimental design method frequently used and recommended to evaluate the effect of guidelines or other complex interventions. ${ }^{9}$

We selected all primary reports of randomised trials indexed in PubMed (publication type "Randomized Controlled Trial") and published in one of five high impact general medical journals (the Annals of Internal Medicine, BMJ, Lancet, JAMA, and New England Journal of Medicine). We excluded reports that did not include an electronic abstract, and any secondary trial publications or economic analyses. Because the number of randomised trials published by journals varies each year, we selected at random (www.random.org) a sample of up to 60 trial reports per journal per year for inclusion in our final sample. If fewer than 60 reports of randomised trials were obtained for a journal in a particular year, we assessed all eligible reports.

\section{Interventions}

We aimed to assess the effect of the publication of the CONSORT for Abstracts guidelines and the effect of different editorial policies used to implement these guidelines. Therefore, we checked each journal's instructions to authors in January 2010 for any reference to the CONSORT for Abstracts guidelines (for example, reference to a publication or link to the relevant section of the CONSORT website). For those journals that mentioned the guidelines in their instructions to authors, we contacted the editor of that journal to ask when the guidance was added, whether the journal enforced the guidelines, and if so, how. We classified journals in three categories: those not mentioning the CONSORT guidelines in their instructions to authors (JAMA and the New England Journal of Medicine); those referring to the guidelines in their instructions to authors, but with no specific policy to implement them $(B M J)$; and those referring to the guidelines in their instructions to authors, with a policy to implement them (Annals of Internal Medicine and the Lancet). In the final journal group, we defined such an implementation policy as having any action to enforce the adherence to CONSORT - that is, an email was sent to authors to revise the abstract according to the CONSORT for Abstracts guidance at the revision stage of the manuscript, or changes were made by the assistant editors of these journals towards the end of the editorial process.

\section{The study period}

For the purpose of the study, we selected abstracts published between January 2006 and December 2009. The CONSORT for Abstracts guidelines were published in the Lancet ${ }^{8}$ and PloS
Medicine $^{7}$ in January 2008 (26 and 22 January, respectively). Shortly after publication (January 2008), the Annals of Internal Medicine, Lancet, and $B M J$ added the guidelines to their instructions to authors.

Overall, the study period had 48 monthly intervals: 25 before the publication of the CONSORT for Abstracts guidelines (January 2008), three during a transition period to accommodate a gradual implementation (February 2008 to April 2008), and 20 after the intervention (we considered that the intervention began in May 2008).

\section{Outcomes}

The primary outcome was a composite outcome. We decided a priori only to focus on items that were reported in fewer than $50 \%$ of the abstracts across the five journals in 2006. By focusing only on items that were poorly reported, we hoped to see the greatest effect of implementation of the guidelines (box, web table 1). Therefore, our primary outcome was the monthly mean number of items reported per abstract on a 0 to 9 scale. For our secondary outcome, we assessed items reported in fewer than $20 \%$ of abstracts across the five journals in 2006 (box, web table 1). Consequently, the secondary outcome was the monthly mean number of items reported per abstract on a 0 to 5 scale.

\section{Data extraction}

For each included abstract, we checked to see whether items included in the CONSORT for Abstracts guidelines were adequately reported or not reported. Single data extraction was carried out by two authors (SH and IB). Abstracts were allocated at random to the two assessors across the five journals and time periods; thus, each assessor reviewed a similar number of abstracts from each journal per year. However, both authors first piloted the data extraction form to ensure consistency in the extraction process. Any uncertainty regarding a particular abstract was checked and resolved by discussion. It was not possible to blind assessors to the journal being reviewed, because abstracts for each journal had their own particular house style.

\section{Data analysis}

We plotted outcomes graphically over time (by month blocks) from 2006 to 2009 (we excluded the three month transition period from the statistical models but included it in the visual presentation of figures $1 \Downarrow$ and $2 \Downarrow$. This allowed us to assess, for the primary and secondary outcomes, any improvements before and after the intervention. We fitted a time series segmented linear regression and estimated monthly use to evaluate changes after the publication of the CONSORT for Abstracts guidelines. The model included terms to evaluate the following variables: a constant term (for levels before the intervention at baseline), a term for linear trends before the intervention, terms to estimate level and trend changes after the intervention, and a residual term. Web appendix 1 provides more information about the statistical model. The ordinary least square model assumes a linear association between time (before and after intervention) and the outcome. We used the Durbin-Watson test to check for autocorrelation. If a Durbin-Watson test result was significant, we corrected the model for autocorrelated errors. One key assumption of ordinary regression analysis is that the errors are independent of each other. To overcome this problem, we used a stepwise autoregression method that initially fits a high order model with many autoregressive lags and then sequentially removes autoregressive parameters until all remaining autoregressive parameters have significant $t$ tests. 


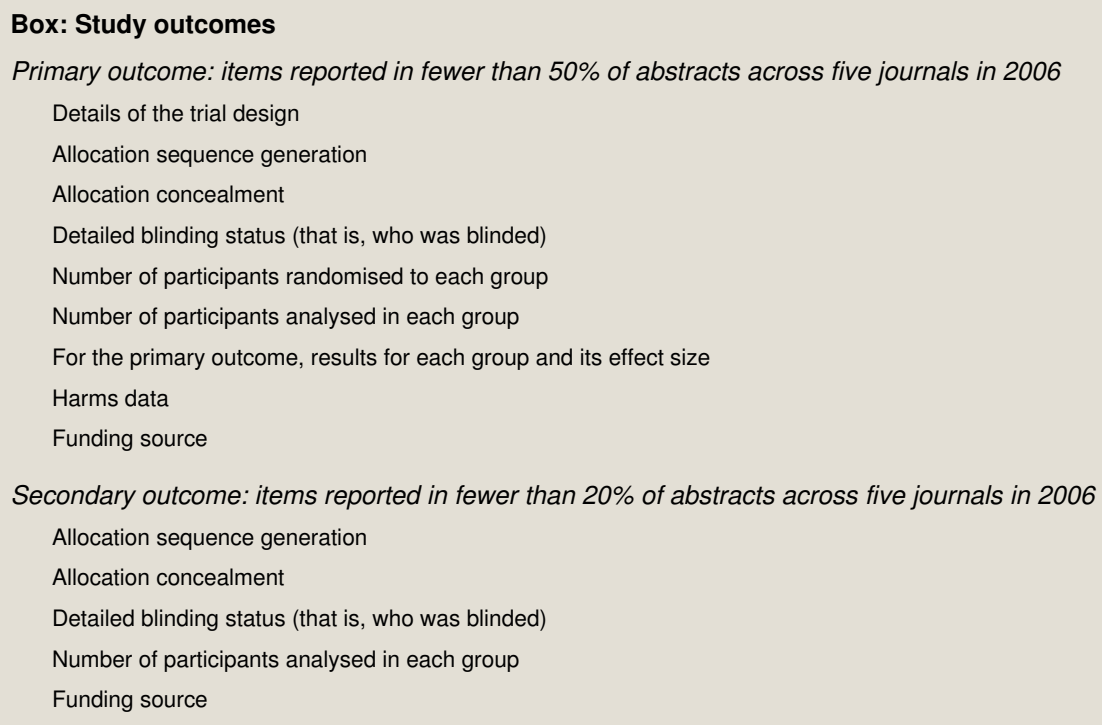

The models (one per outcome) used to estimate within group changes were built on each of the following samples: all journals, journals not mentioning the CONSORT for Abstracts guidelines in their instructions to authors, journals referring to the guidelines in their instructions to authors but with no policy to implement them, and journals referring to the guidelines in their instructions to authors with a policy to implement them.

Finally, we added a dummy group variable to indicate an active or inactive editorial policy and bivariate interaction terms (that is, a group by pre-intervention trend, a group by change in level, and a group by change in trend) to the initial models (one per outcome), to test for differences between journals with an active policy for implementation (Annals of Internal Medicine, Lancet) and those with no active policy (BMJ, JAMA, New England Journal of Medicine). We used SAS statistical software (release 9.2, SAS Institute) for the statistical analyses, and considered $\mathrm{P}<0.05$ as significant.

\section{Results}

We included 955 reports of abstracts of randomised trials in our final sample and included trials from a wide variety of clinical specialties.

\section{All journals}

Figure 1 and web table 2 detail the change in the mean number of items reported per abstract for the primary and secondary outcomes before and after the introduction of the CONSORT for Abstracts guidelines for all journals. Overall, for the primary outcome, publication of the CONSORT guidelines did not lead to a significant increase in the level of the mean number of items reported (increase of 0.3035 of nine items, $\mathrm{P}=0.16$ ) or the trend (increase of 0.0193 items per month, $\mathrm{P}=0.21$ ). For the secondary outcome, we saw a significant increase in the level of the mean number of items reported after the implementation of the CONSORT guidelines (increase of 0.3882 of five items, $\mathrm{P}=0.0072$ ) and in trends (increase of 0.0288 items per month, $\mathrm{P}=0.0025)$. Web table 3 provides the individual data per checklist item, per journal per year.

\section{Effect of the journal policy}

We first focused on journals not mentioning the CONSORT for Abstracts guidelines in their instructions to authors (JAMA, New England Journal of Medicine) and journals referring to the guidelines in their instructions to authors but with no policy of enforcement of them $(B M J)$. We observed no significant increase in the level change or trend change for the primary and secondary outcomes (fig 2, web table 2).

However, when focusing on journals with an active policy to enforce the CONSORT guidelines (Annals of Internal Medicine, Lancet), the publication and editorial policy led to an immediate increase in the level of mean number of items reported but no further improvement in trend for the primary outcome (fig 2, web table 2). At baseline, in January 2006, the mean number of items reported per abstract was 1.52 of nine items, which increased to 2.56 nine items during the 25 months before the intervention. Implementation of the CONSORT guidelines led to an immediate significant increase of 1.50 immediately after the intervention $(\mathrm{P}=0.0037)$. However, the trend did not change (increase of 0.0193 items per month, $\mathrm{P}=0.6$ ). In December 2009, 23 months after the publication of the guidelines, the mean number of items reported per abstract for the primary outcome in the Annals of Internal Medicine and the Lancet was 5.41 items, which represented a $53 \%$ increase compared with the expected level estimated on the basis of pre-intervention trends. Web appendix 2 shows data for each of the nine items, per journal, over time.

For the primary outcome, we used a model to compare journals that had an active policy with those that did not have an active policy. The model showed a significant difference in the change in level (that is, relative to the inactive policy group). The change in level immediately after implementation of the CONSORT for Abstracts guidelines increased significantly (increase of 1.90 items, $\mathrm{P}=0.0006$ for interaction test). However, we saw a non-significant difference in trend change (increase of 0.0316 items per month relative to the inactive policy group, $\mathrm{P}=0.3915$ for interaction test).

For the secondary outcome, when focusing only on the Annals of Internal Medicine and Lancet at baseline in January 2006, the mean number of items reported per abstract was 0.18 of five items (fig 2, web table 2). This level had increased to 0.42 items during the 25 months before the intervention. Implementation of the CONSORT for Abstracts guidelines led to a significant increase in the mean number of items reported per abstract immediately after the intervention (increase of 1.03 items, $\mathrm{P}=0.0035$ ) and a significant change in trend (increase of 0.0613 items per month, $\mathrm{P}=0.0154)$. In December 2009, 23 months after publication of the guidelines, the secondary outcome 
reached 2.91 points in the Annals of Internal Medicine and Lancet, a 4.5-fold (350\%) increase compared with the expected level estimated on the basis of pre-intervention trends.

The model used for comparing journals that had an active policy with those that did not have active policy for the secondary outcome showed a significant difference between groups in the change in level (relative to the inactive policy group, increase of 1.24 items, $\mathrm{P}<0.0001$ for interaction test) and change in trend after implementation of the CONSORT for Abstracts guidelines (increase of 0.0761 items per month, $\mathrm{P}<0.0001$ for interaction test).

\section{Discussion}

This study evaluated the effect of journals' editorial policy to implement the CONSORT extension for abstracts guidelines on the reporting quality of abstracts of randomised trials published in five high impact, general medical journals. After publication of the guidelines in January 2008, we identified a significant increase in the reporting of key items in the two journals (Annals of Internal Medicine, and Lancet) that endorsed the guidelines in their instructions to authors and that had an active editorial policy to implement them. By comparison, we observed no significant difference in the one journal $(B M J)$ that endorsed the guidelines but did not have an active

implementation strategy, and in the two journals (JAMA, New England Journal of Medicine) that did not endorse the guidelines in their instructions to authors.

\section{Comparison with other studies}

Several studies have assessed the quality of published reports of abstracts of randomised trials. ${ }^{2-4}$ 10-12 These studies all highlight problems in the accuracy and quality of abstract reporting, particularly a lack of information about the trial methodology and the robustness of its results. ${ }^{21}$ No studies have evaluated the effect of the CONSORT for Abstracts guidelines over time. Our study used a segmented regression of interrupted time series analyses, a quasi-experimental design method to evaluate the effect of the guidelines. ${ }^{13}$ In such designs, multiple measurements are taken before and after an intervention are applied. This design is effective if a fairly large number of measurements for the outcome of interest are available and a segmented regression can be used to analyse the data. Segmented regression can identify both sudden change due to an intervention, as well as more gradual changes over time.

For clinical practice guidelines, reporting guidelines aim to improve knowledge (authors are aware of the guidelines), attitudes (authors agree and accept the guidelines as a new standard), behaviour (authors change practice patterns to conform with the guidelines), and outcomes (the guidelines improve reporting).$^{14}$ For this purpose, a strategy of dissemination and implementation of the guidelines is necessary. $^{15}$

The CONSORT for Abstract guidelines were disseminated through its publication in two general medical journals with high impact factors. ${ }^{78}$ In October 2008, the International Committee of Medical Journal Editors also recommended that "articles on clinical trials should contain abstracts that include the items that the CONSORT group has identified as essential (www.consort-statement.org)" (www.icmje.org). Furthermore, since publication, the guidelines have also been incorporated into the recently revised main CONSORT statement in March $2010 .{ }^{16}{ }^{17}$ Dissemination was also enforced by some journal editors referring to the CONSORT for Abstracts guidelines in their instructions to authors.
However, our results show that the guidelines improved reporting only when actively implemented by a specific editorial policy. This policy implied a relatively simple intervention consisting of an email sent to authors to revise the abstract according to CONSORT guidance at the revision stage of the manuscript and, in some instances, further changes made by the journals' assistant editors at the end of the editorial process. Our results accord with those of systematic reviews showing that, in various fields, passive dissemination of information was generally ineffective and that the use of specific strategies to implement research based recommendations seems to be necessary to ensure that practices change. ${ }^{18}$

\section{Limitations}

Our study has several limitations. We based our study on abstracts of randomised trials published in five high impact, general medical journals, all of which have considerable resources to support their work. We recognise that the resources and procedures available for these journals might not be the same for all journals; therefore, it is not possible to say that what works in these journals is applicable in other smaller, less well resourced journals. Furthermore, the overall reporting quality of abstracts published in these five journals might be higher than in other less well known journals, which was certainly the case when comparing the reporting of CONSORT items in general journals with reporting in specialty journals. ${ }^{19}$

Our primary outcome was a composite outcome, which focused on the nine CONSORT items reported in fewer than $50 \%$ of abstracts in 2006. This composite outcome assumed that each of the nine items were equally important, which might not always be the case, depending on the perspective of the reader. However, by focusing only on items that were poorly reported, we hoped to see the greatest effect of implementation of the guidelines.

Finally, we allowed a three month transition period in our study, during which we could implement and measure the effect of the CONSORT for Abstracts guidelines. This transition period might have been longer for some articles, since they can take longer than three months to pass through the editorial process.

We thank Cindy Mulrow (Annals of Internal Medicine), Trish Groves (BMJ), and David McNamee (Lancet) for providing an insight into their journal editorial processes, and all those involved in development of the CONSORT for Abstracts guidelines.

Contributors: SH and IB were involved in the design, implementation, and analysis of the study; and in writing the final manuscript. PR and GB were involved in the design and analysis of the study, and in commenting on drafts of the final manuscript. $\mathrm{SH}$ is responsible for the overall content as guarantor.

Funding: This study received no external funding.

Competing interests: All authors have completed the Unified Competing Interest form at www.icmje.org/coi_disclosure.pdf (available on request from the corresponding author) and declare: no support from any organisation for the submitted work; no financial relationships with any organisations that might have an interest in the submitted work in the previous three years; $\mathrm{SH}, \mathrm{IB}$, and PR are members of the CONSORT Group; no other relationships or activities that could appear to have influenced the submitted work.

Data sharing: Technical appendix, statistical code, and dataset available from the corresponding author at: sally.hopewell@csm.ox.ac.uk.

[No authors listed.] The impact of open access upon public health. PLoS Med 2006;3:e252. Berwanger O, Ribeiro RA, Finkelsztejn A, Watanabe M, Suzumura EA, Duncan BB, et al. The quality of reporting of trial abstracts is suboptimal: survey of major general medical journals. J Clin Epidemiol 2009;62:387-92. 


\section{What is already known on this topic}

Problems in abstract accuracy and quality include a lack of information about trial methods and the robustness of results; such inadequate reporting could seriously mislead a reader's interpretation of trial findings

The CONSORT for Abstract guidelines were published in 2008, listing essential items to include when reporting the main results of a randomised trial in an abstract

The effect of these guidelines has not been widely evaluated

\section{What this study adds}

Based on results from five high impact medical journals, endorsement and active implementation of the CONSORT for Abstracts guidelines by journal editors can lead to improvements in the reporting of abstracts of randomised trials

Although authors bear the main responsibility for implementing the guidelines, journals can, and should, have an important role in implementing them

3 Hopewell S, Eisinga A, Clarke M. Better reporting of randomized trials in biomedical journal and conference abstracts. $J$ Info Sci 2007;34:162-73.

4 Pitkin RM, Branagan MA, Burmeister LF. Accuracy of data in abstracts of published research articles. JAMA 1999;281:1110-1.

5 Froom P, Froom J. Deficiencies in structured medical abstracts. J Clin Epidemio 1993:46:591-4.

6 loannidis JP, Lau J. Completeness of safety reporting in randomized trials: an evaluation of 7 medical areas. JAMA 2001;285:437-43.

7 Hopewell S, Clarke M, Moher D, Wager E, Middleton P, Altman DG, et al. CONSORT for reporting randomized controlled trials in journal and conference abstracts: explanation and elaboration. PLoS Med 2008;5:e20.

8 Hopewell S, Clarke M, Moher D, Wager E, Middleton P, Altman DG, et al. CONSORT for reporting randomised trials in journal and conference abstracts. Lancet 2008;371:281-3.

9 Wagner AK, Soumerai SB, Zhang F, Ross-Degnan D. Segmented regression analysis of interrupted time series studies in medication use research. $J$ Clin Pharm Ther 2002;27:299-309.

10 Chen Y, Li J, Ai C, Duan Y, Wang L, Zhang M, et al. Assessment of the quality of reporting in abstracts of randomized controlled trials published in five leading Chinese medical journals. PLoS One 2010;5:e11926.

11 Can OS, Yilmaz AA, Hasdogan M, Alkaya F, Turhan SC, Can MF, et al. Has the quality of abstracts for randomised controlled trials improved since the release of Consolidated Standards of Reporting Trial guideline for abstract reporting? A survey of four high-profile anaesthesia journals. Eur J Anaesthesiol 2011;28:485-92.

12 Yoon U, Knobloch K. Quality of reporting in sports injury prevention abstracts according to the CONSORT and STROBE criteria: an analysis of the World Congress of Sports Injury Prevention in 2005 and 2008. Br J Sports Med 2012;46:202-6.

13 Cook TD, Campbell DT. Quasi-experiments: interrupted time-series designs in quasi-experimentation: design and analysis issues for field settings. Houghton Mifflin Company, 1979
14 Woolf SH. Practice guidelines: a new reality in medicine. III. Impact on patient care. Arch Intern Med 1993;153:2646-55.

15 Cabana MD, Rand CS, Powe NR, Wu AW, Wilson MH, Abboud PA, et al. Why don't physicians follow clinical practice guidelines? A framework for improvement. JAMA 1999;282:1458-65

16 Moher D, Hopewell S, Schulz KF, Montori V, Gotzsche PC, Devereaux PJ, et al. CONSORT 2010 explanation and elaboration: updated guidelines for reporting parallel group randomised trials. BMJ 2010;340:c869.

17 Schulz KF, Altman DG, Moher D. CONSORT 2010 statement: updated guidelines for reporting parallel group randomised trials. BMJ 2010;340:c332.

18 Bero LA, Grilli R, Grimshaw JM, Harvey E, Oxman AD, Thomson MA. Closing the gap between research and practice: an overview of systematic reviews of interventions to promote the implementation of research findings. The Cochrane Effective Practice and Organization of Care Review Group. BMJ 1998;317:465-8.

19 Mills E, Wu P, Gagnier J, Heels-Ansdell D, Montori VM. An analysis of general medical and specialist journals that endorse CONSORT found that reporting was not enforced consistently. J Clin Epidemiol 2005;58:662-7.

Accepted: 17 April 2012

\section{Cite this as: BMJ 2012;344:e4178}

This is an open-access article distributed under the terms of the Creative Commons Attribution Non-commercial License, which permits use, distribution, and reproduction in any medium, provided the original work is properly cited, the use is non commercial and is otherwise in compliance with the license. See: http://creativecommons.org/licenses/by$\mathrm{nc} / 2.0 /$ and http://creativecommons.org/licenses/by-nc/2.0/legalcode. 


\section{Figures}
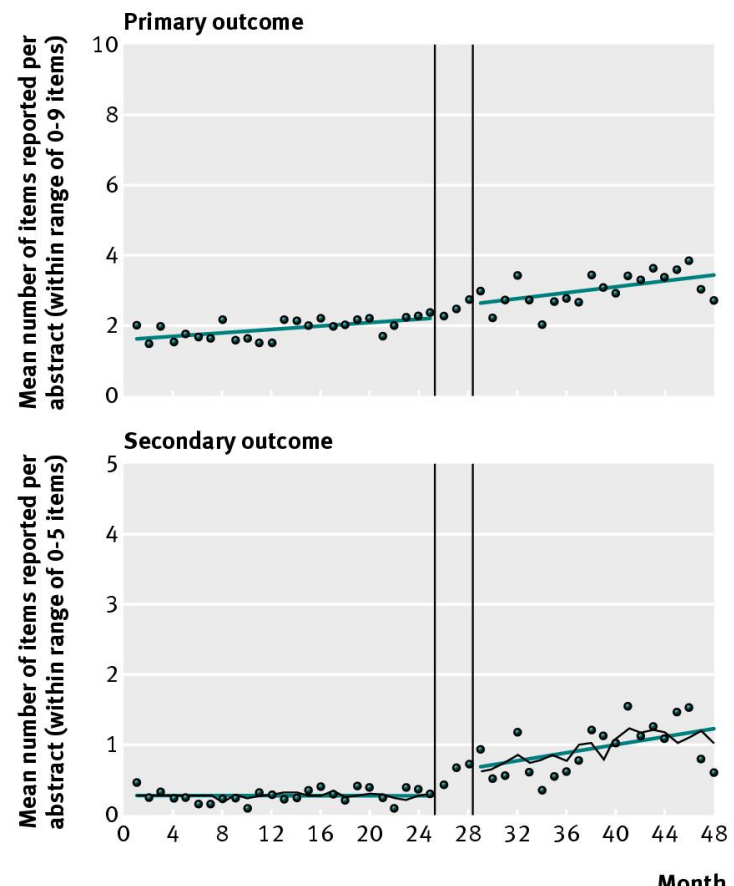

Fig 1 Change in outcomes from January 2006 to December 2009, before and after the introduction of the CONSORT for Abstracts guidelines implemented in January 2008 for all journals. Circles=actual values; straight lines=regression lines traced out by the structural predicted values; jagged lines=full model prediction values formed from both the structural and autoregressive parts of the model; vertical lines=transition phase (February to April 2008) 
Primary outcome

Secondary outcome

CONSORT guidelines included in journal's instructions to authors, with active implementation policy (Annals of Internal Medicine, Lancet)

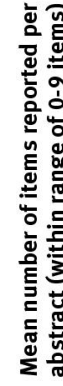

4
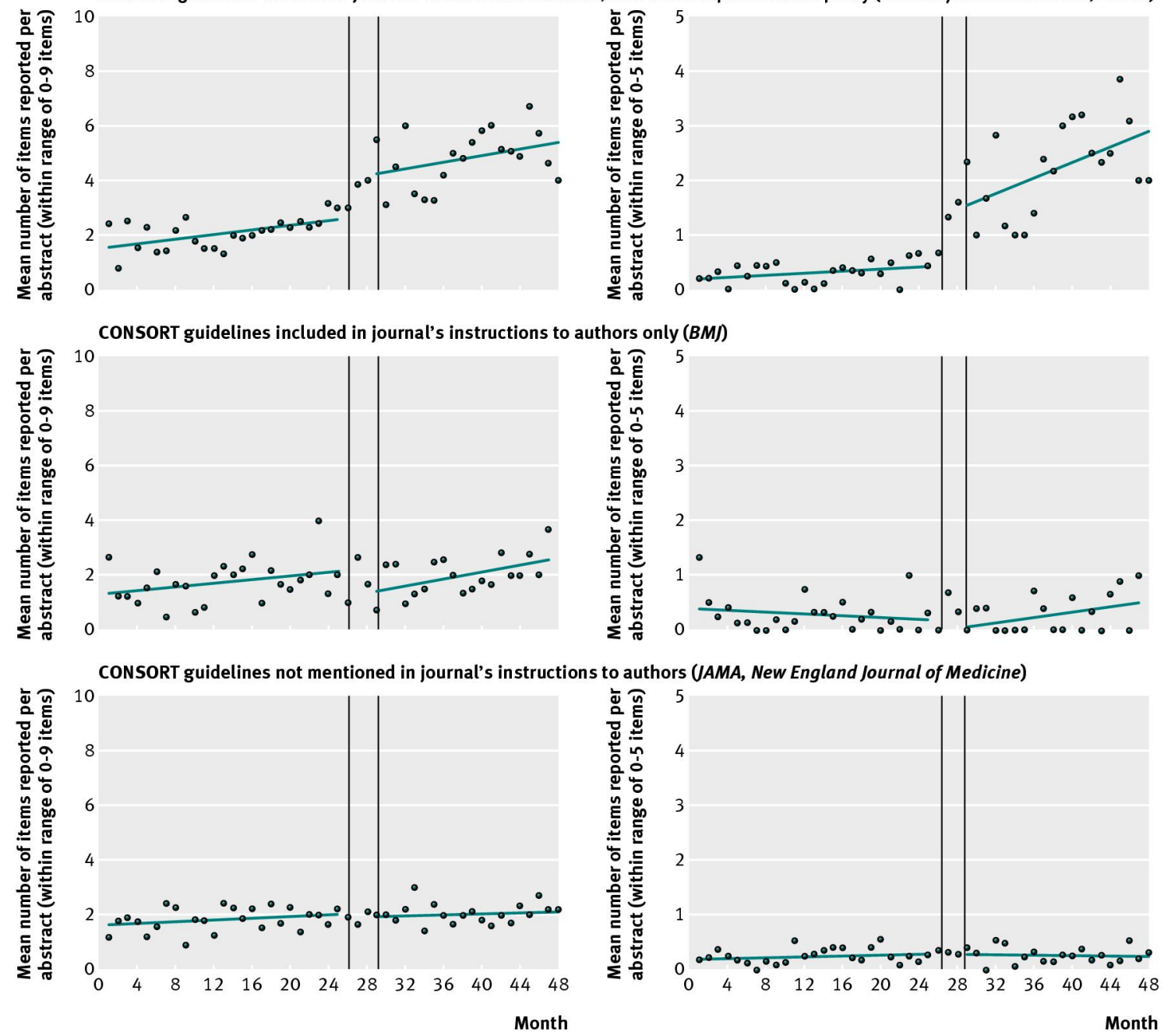

Fig 2 Change in outcomes from January 2006 to December 2009, before and after introduction of the CONSORT for Abstracts guidelines implemented in January 2008 for each journal category. Circles=actual values; straight lines=regression lines traced out by the structural predicted values; vertical lines=transition phase (February to April 2008) 